\title{
Future Perspectives on Baryon Form Factor Measurements with BES III
}

\author{
Karin Schönning ${ }^{1, a}$ and Cui $\mathrm{Li}^{1}$ \\ ${ }^{1}$ Department of Physics and Astronomy, Uppsala University, Box 516, S-75120 Uppsala, Sweden
}

\begin{abstract}
The electromagnetic structure of hadrons, parameterised in terms of electromagnetic form factors, EMFF's, provide a key to the strong interaction. Nucleon EMFF's have been studied rigorously for more than 60 years but the new techniques and larger data samples available at modern facilities have given rise to a renewed interest for the field. Recently, the access to hyperon structure by hyperon time-like EMFF provides an additional dimension. The BEijing Spectrometer (BES III) at the Beijing Electron Positron Collider (BEPC-II) in China is the only running experiment where time-like baryon EMFF's can be studied in the $e^{+} e^{-} \rightarrow B \bar{B}$ reaction. The BES III detector is an excellent tool for baryon form factor measurements thanks to its near $4 \pi$ coverage, precise tracking, PID and calorimetry. All hyperons in the SU(3) spin $\frac{1}{2}$ octet and spin $\frac{3}{2}$ decuplet are energetically accessible within the BEPC-II energy range. Recent data on proton and $\Lambda$ hyperon form factors will be presented. Furthermore, a world-leading data sample was collected in 2014-2015 for precision measurements of baryon form factors. In particular, the data will enable a measurement of the relative phase between the electric and the magnetic form factors for $\Lambda$ and $\Lambda_{c}^{+}$and hyperons. The modulus of the phase can be extracted from the hyperon polarisation, which in turn is experimentally accessible via the weak, parity violating decay. Furthermore, from the spin correlation between the outgoing hyperon and antihyperon, the sign of the phase can be extracted. This means that the time-like form factors can be completely determined for the first time. The methods will be outlined and the prospects of the BES III form factor measurements will be given. We will also present a planned upgrade of the BES III detector which is expected to improve future form factor measurements.
\end{abstract}

\section{Introduction}

One of the most challenging questions in contemporary physics is why and how quarks are confined into hadrons. The nucleon provides a key to understand the strong interaction between the quarks. Nucleons constitute the major part of the mass of the visible universe and are the simplest system for which the non-abelian nature of the strong interaction is manifest [1]. However, despite a century of rigorous research, several aspects of the nucleon remain a puzzle. For example, we neither understand the mass nor the spin of the nucleon: the current quarks only constitute $\approx 99 \%$ of the total nucleon mass and $\approx 33 \%$ if its spin. These challenges, often referred to as the mass generation problem and the spin crisis, respectively, are non-perturbative effects of the strong interaction and belong to

ae-mail: karin.schonning@physics.uu.se 
the most intriguing questions in physics. Important insights about how the quarks form nucleons can be obtained by studying the electromagnetic structure. Space-like Electromagnetic Form Factors (EMFF's) are hadron structure observables that, in the case of the proton, have been studied rigorously since the 1960's [2] in elastic electron-proton scattering. The polarimeter techniques developed in the 1990's contributed to a revival of the field and recently, numerous electron-positron colliders have given access also to the time-like region. The large data bank of proton form factors has inspired the theory community which resulted in new insights regarding non-perturbative strong interactions [3].

An additional angle to nucleon structure is provided by hyperons. The key question is: "What happens if you replace one of the light quarks in a nucleon, with a heavier one?". If SU(3) flavour symmetry were exact, the properties of a hyperon could be derived from those of the nucleon. Observed deviations therefore provide a measurement of the extent to which SU(3) symmetry is broken. The scale of the strong interaction probed in a measurement is governed by the mass of the quarks in the produced system. In the case of strange hyperons, the scale is set by the mass of the strange quark, i.e. $m_{s} \approx 100 \mathrm{MeV} /$. This is close to the QCD cut-off $\Lambda_{Q C D} \approx 200 \mathrm{MeV}$ which corresponds to the confinement regime of the strong interaction. The charm quark is more than ten times heavier, i.e. $m_{c} \approx 1300 \mathrm{MeV}$ which corresponds to the scale just where perturbative QCD breaks down. Thus, charmed and strange hyperons probe two different regions of the strong interactions and enable a step-wise extension of existing theories into regions which until now are poorly understood.

Hyperons are difficult to study in the space-like region since they are unstable - hyperon targets are unfeasible and hyperon the quality of hyperon beams is in general not sufficient. Time-like form factors therefore offers the best opportunity to study hyperon structure. Recent technological advances regarding high luminosity beams and dedicated, precise detectors open up new possibilities for nucleon and hyperon structure studies. Hyperon structure is however rather unexplored territory.

\section{Formalism of time-like form factors}

Consider the production of a spin $\frac{1}{2}$ baryon-antibaryon pair in $e^{+} e^{-} \rightarrow B \bar{B}$ where the baryon can be either a nucleon or a hyperon. Assuming that one-photon exchange (OPE) $e^{+} e^{-} \rightarrow \gamma * \rightarrow B \bar{B}$ is the dominating process, then the differential cross section can be parameterised in terms of electric and magnetic form factors $G_{E}$ and $G_{M}$. These form factors are assumed to be analytical functions of the momentum transfer squared $q^{2}$ :

$$
\frac{d \sigma_{B o r n}\left(q^{2}\right)}{d \Omega}=\frac{\alpha^{2} \beta C}{4 q^{2}}\left[\left|G_{M}\left(q^{2}\right)\right|^{2}\left(1+\cos ^{2} \theta_{B}\right)+\frac{1}{\tau}\left|G_{E}\left(q^{2}\right)\right|^{2}\left(\sin ^{2} \theta_{B}\right)\right] .
$$

Here, $\alpha \approx \frac{1}{137}$ is the fine structure constant and $\theta_{B}$ the scattering angle of the baryon. The $\beta$ and $\tau$ are kinematic factors: $\beta=\frac{v}{c}, v$ being the velocity of the outgoing baryon, and $\tau=\frac{q^{2}}{4 m_{B}^{2}}, m_{B}$ being the mass of baryon. The Coulomb factor $C$ accounts for the electromagnetic $B \bar{B}$ interactions of point-like baryons and is equal to 1 for neutral baryons and $\frac{\pi \alpha}{\beta} \frac{1}{1-\exp (-\pi \alpha / \beta)}$ for charged baryons.

Integrating over the full solid angle, the total cross section is obtained:

$$
\sigma_{\text {Born }}\left(q^{2}\right)=\frac{4 \pi \alpha^{2} \beta C}{3 q^{2}}\left[\left|G_{M}\left(q^{2}\right)\right|^{2}+\frac{1}{2 \tau}\left|G_{E}\left(q^{2}\right)^{2}\right|\right] .
$$

From the total cross section, the effective form factor $G\left(q^{2}\right)$ can be defined [4]:

$$
\left|G\left(q^{2}\right)\right|^{2}=\frac{2 \tau\left|G_{M}\left(q^{2}\right)\right|^{2}+\left|G_{E}\left(q^{2}\right)\right|^{2}}{2 \tau+1}=\frac{\sigma_{B o r n}\left(q^{2}\right)}{\left(1+\frac{1}{2 \tau}\right)\left(\frac{4 \pi \alpha^{2} \beta C}{3 q^{2}}\right)}
$$


It is often convenient to describe the production in terms of the effective form factor and the ratio $R=\left|G_{E}\left(q^{2}\right) / G_{M}\left(q^{2}\right)\right|$ between the electric and the magnetic form factor. The ratio $R$ is extracted from the angular distribution of the outgoing baryons.

Time-like EMFF's are complex with a relative phase $\Delta \Phi$. This phase induces polarisation effects even if the initial state is unpolarised. In strong and electromagnetic interactions, where parity is conserved, the polarisation can be non-zero perpendicular to the production plane spanned by the incoming beam and the outgoing baryon or antibaryon, as shown in Fig. 1. The polarisation axis is denoted $n$ in the figure.

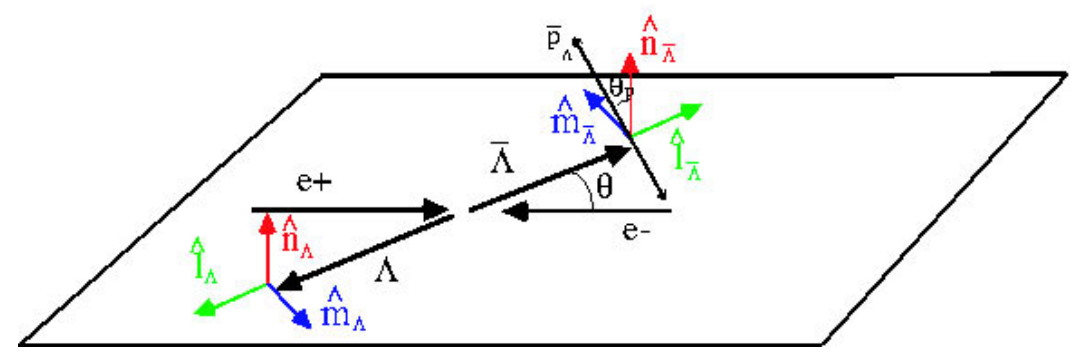

Figure 1. The reference system of the $e^{+} e^{-} \rightarrow \gamma * \rightarrow \Lambda \bar{\Lambda}$ reaction.

The polarisation depends on the phase $\Delta \Phi$ and the scattering angle of the baryon in the following way [5]:

$$
P_{n}=-\frac{\sin 2 \theta_{B} / \sqrt{\tau}}{\left(1+\cos ^{2} \theta_{B}\right)+\frac{R^{2}}{\tau}\left(1-\cos ^{2} \theta_{B}\right)} R \sin \Delta \Phi
$$

Therefore, $\sin \Delta \Phi$, and thus the modulus of the phase, can be extracted from the hyperon polarisation, as illustrated to the left in figure 2. Furthermore, the spin correlation of the outgoing hyperon and antihyperon depends on $\cos \Delta \Phi$ :

$$
C_{m l}=\frac{\sin 2 \theta_{B} / \sqrt{\tau}}{\left(1+\cos ^{2} \theta_{B}\right)+\frac{R^{2}}{\tau}\left(1-\cos ^{2} \theta_{B}\right)} \operatorname{Ros} \Delta \Phi
$$

This is shown in the right panel of figure 2. Hyperons have an advantage to protons in the sense that they decay weakly. The decay of a spin $\frac{1}{2}$ hyperon $Y$ into a spin $\frac{1}{2}$ baryon, in the following denoted $p$, and a spin 0 meson has one parity conserving amplitide and one parity violating part. Their interference causes the daugther baryon to be emitted in the direction of the polarisation of the mother hyperon, according to

$$
I\left(\theta_{p}\right) \propto\left(1+\alpha_{Y} P_{n} \cos \theta_{p}\right)
$$

where $\theta_{p}$ is the angle between the decay baryon in the hyperon rest frame and the polarisation axis of the hyperon. The $\alpha_{Y}$ is the so-called asymmetry parameter and is related to the parity violating and parity conserving amplitudes of the decay. Thus, the polarisation of hyperons is accessible via the angular distribution of their decay. The spin correlations $C_{i j}$ are accessible via

$$
I\left(\theta_{p}, \theta_{\bar{p}}\right) \propto\left(1+\alpha_{Y} \alpha_{\bar{Y}} C_{i j} \cos \theta_{i p} \cos \theta_{\bar{j}}\right) .
$$



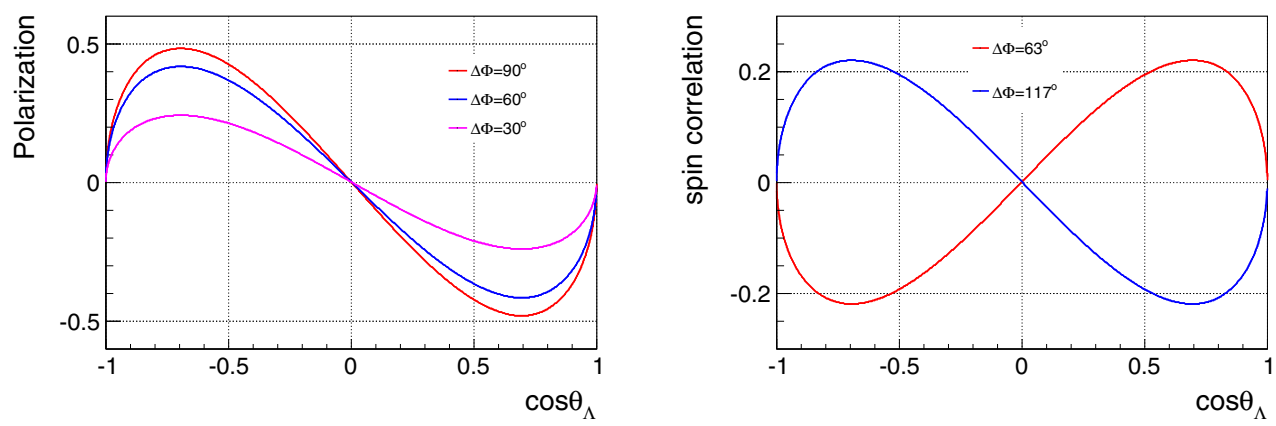

Figure 2. Left: The polarisation $P_{n}$ as a function of the hyperon scattering angle $\cos \theta_{\Lambda}$ for $R=\left|G_{E} / G_{M}\right|=1$ and for different values of the modulus of the phase $|\Delta \Phi|$ between the electric and the magnetic form factor. Right: The spin correlation $C_{x z}$ as a function of the hyperon scattering angle $\cos \theta_{\Lambda}$ for $\Delta \Phi=63^{\circ}$ and for $\Delta \Phi=180^{\circ}-63^{\circ}=117^{\circ}$.

Thus, in case of exclusive hyperon production, both polarisation and spin correlations can be measured. Since this implies that the modulus as well as the sign of the phase are accessible, the EMFF's can be completely determined.

\section{The BES III experiment}

The measurements will be carried out with the Beijing Spectrometer (BESIII) [6] at the Beijing Electro Positron Collider (BEPCII) in Beijing, China. The BEPCII is optimised in the $\tau$-charm energy region but after recent upgrades, the energy range has been extended down to CMS energies of $2 \mathrm{GeV}$. This means that hadron form factors can be accessed with two different methods: i) by direct $e^{+} e^{-} \rightarrow$ $\gamma^{*} \rightarrow h \bar{h}$ production in an off-resonance energy scan and ii) by the ratiative return method [7] in $e^{+} e^{-} \rightarrow e^{+} e^{-} \gamma_{I S R} \rightarrow \gamma^{*} \gamma_{I S R} \rightarrow h \bar{h} \gamma_{I S R}$ using a large data sample collected at some charmonium resonance, e.g $\Psi^{\prime}(3770)$. The BESIII detector has an geometrical acceptance of $93 \%$ of the $4 \pi$ solid angle. It contains a small-celled, helium-based main drift chamber (MDC) providing momentum measurements of charged particles; a time-of-flight system (TOF) based on plastic scintillator for identification of charged particles; an electromagnetic calorimeter (EMC) made of CsI (Tl) crystals for triggering and measurement of the energies of photons; a muon system (MUC) made of Resistive Plate Chambers (RPC); a super-conductive solenoid magnet with a central field of 1.0 Tesla. The momentum resolution of the charged particles is $0.5 \%$ at $1.0 \mathrm{GeV} / c$ in a 1.0 Tesla magnetic field. The energy loss $(d \mathrm{E} / d x)$ measurement provided by the MDC has a resolution better than $6 \%$ for electrons from Bhabha scattering. For $1.0 \mathrm{GeV}$ photon, the energy resolution can reach $2.5 \%$ (5\%) in the barrel (endcaps) of the EMC, and the position resolution is $6 \mathrm{~mm}$ in the barrel and $9 \mathrm{~mm}$ in the endcaps. The time resolution of TOF is $80 \mathrm{ps}$ in the barrel and $110 \mathrm{ps}$ in the endcaps, corresponding to a $2 \sigma K / \pi$ separation for momenta up to about $1.0 \mathrm{GeV} / c$. The spatial resolution of MUC is better than $2 \mathrm{~cm}$.

\section{Recent results by BES III}

In 2011-2012, a pilot energy scan was performed where data were collected at 12 different energies between $2.2324 \mathrm{GeV}$ and $3.671 \mathrm{GeV}$. This enabled a measurement of the proton effective form factor [8]. In addition, the ratio between the electric and the magnetic form factors was measured at three 
energies: $2.4 \mathrm{GeV}, 2.8 \mathrm{GeV}$ and $3.065 \mathrm{GeV}$. The results, shown in figure 3 and figure 4, agree with previous data from e.g BaBar [4, 9-13] and improved the precision with about 30\%. Furthermore, the ratio $\left|G_{E} / G_{M}\right|$ was extracted in three data points. At four energies, i.e. at $2.2324 \mathrm{GeV}, 2.4 \mathrm{GeV}$, $2.8 \mathrm{GeV}$ and $3.065 \mathrm{GeV}$, the samples were sufficiently large to extract the effective form factor of the $\Lambda$ hyperon. The $2.2324 \mathrm{GeV}$ energy point is the closest to the $\Lambda \bar{\Lambda}$ threshold so far. The cross section was measured via two different inclusive final states: the charged $\Lambda \rightarrow p \pi^{-} / \bar{\Lambda} \rightarrow \bar{p} \pi^{+}$and the neutral $\bar{\Lambda} \rightarrow \bar{n} \pi^{0}$, giving consistent results. The combined value of $319.5 \pm 57.6 \mathrm{pb}$ is much larger than expectations using the parameterisation in eq. 1. This shows that the underlying physics is more complicated. Away from threshold, where BES III data overlap with data from BaBar [15], the results are consistent with previous data and the parameterisation in eq. 1 . These preliminary results are shown in Fig. 5 together with previous measurements $[15,16]$.
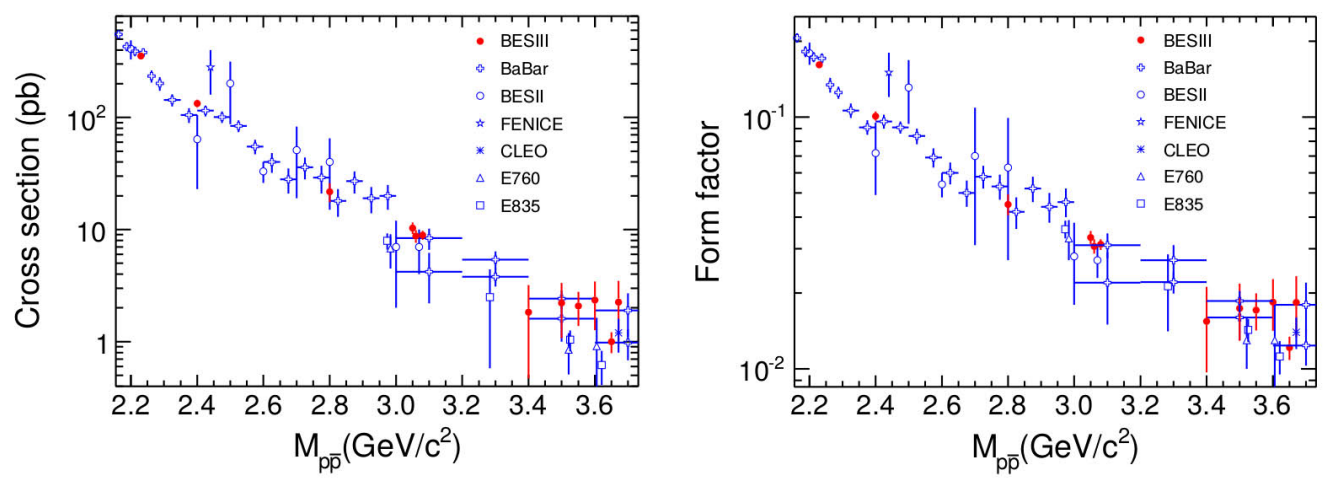

Figure 3. Left: The BESIII cross section of the $e^{+} e^{-} \rightarrow \gamma^{*} \rightarrow p \bar{p}$ process as a function of the CMS energy [8], along with results from other experiments [4, 9-13]. Right: The effective form factor of the proton obtained from the same measurements.

\section{Future perspectives with new data}

New hadron form factor measurements is one of the most important reasons why BES III has collected an unprecedented amount of off-resonance data. The major part was collected in 2014 and 2015 within the CMS energy range 2-3 GeV and above the threshold of the $e^{+} e^{-} \rightarrow \Lambda_{c}^{+} \overline{\Lambda_{c}^{-}}$reaction. The integrated luminosities at the different energies are shown in figure 6 along with the thresholds of different baryon production channels. These data enable a great leap forward regarding baryon structure, since all baryons in the ground state spin $\frac{1}{2} \mathrm{SU}(3)$ octet and spin $\frac{3}{2}$ decuplet are accessible:

- Proton form factors: The expected statistical precision of the effective form factor is between $0.3 \%$ (at $2.125 \mathrm{GeV}$ ) and $13 \%$ (at $2.8 \mathrm{GeV}$ ). Furthermore, the ratio $\left|G_{E} / G_{M}\right|$ can be measured at 16 different energy points. The expected precision of $\left|G_{E} /\right|$ and $\left|G_{M}\right|$ will be similar as the state-of-theart in the space-like region, which enables a first, systematic comparison between space-like and time-like form factors. 

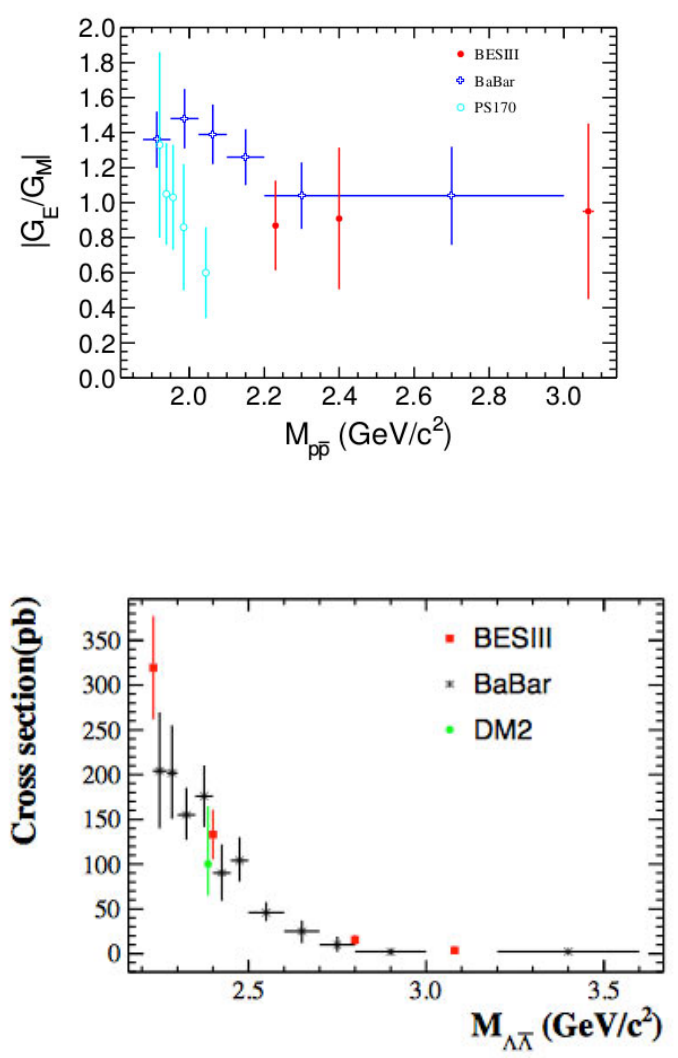

Figure 4. The ratio between the proton electric and magnetic form factor, $\left|G_{E} / G_{M}\right|$, at different energy points. The data are from BESIII [8], BaBar [4] and PS170 [14].

Figure 5. Left: The cross section of the $e^{+} e^{-} \rightarrow \gamma^{*} \rightarrow \Lambda \bar{\Lambda}$ measured by BES III, BaBar [15] and DM2 [16]. Right: The effective form factor of $\Lambda$ exracted from the cross sections to the left.

- Neutron form factors: The effective form factor can be measured with unprecedented precision above $2 \mathrm{GeV}$ and provide the first measurement ever of the ratio $\left|G_{E} / G_{M}\right|$.

- $\Lambda$ hyperon form factors: The data sample enable measurements of the effective form factor and the ratio $\left|G_{E} / G_{M}\right|$ in five energy points. Furthermore, at $2.396 \mathrm{GeV}$, the data sample is sufficient for measurement of the phase $\Delta \Phi$, which means that the form factors can be completely determined for the first time.

- $\Sigma$ hyperon form factors: The effective form factors of the $\Sigma$ triplet can be determined in several points, as well as the ratio of the $\Sigma^{+}$form factors.

- Multi-strange hyperon form factors: Effective form factors can be measured.

- $\Lambda_{c}^{+}$hyperon form factors: The effective form factor can be measured in four pionts, the ratio $\left|G_{E} / G_{M}\right|$ can be determined for the first time in two points and the phase $\Delta \Phi$, and thus the complete elctromagetic form factors, can be determined at $4.6 \mathrm{GeV}$. 
The complete determination of the $\Lambda$ and $\Lambda_{c}^{+}$form factors is particularly interesting since we probe two different energy scales of the strong interaction. The analysis is in progress and the results will be very illuminating.

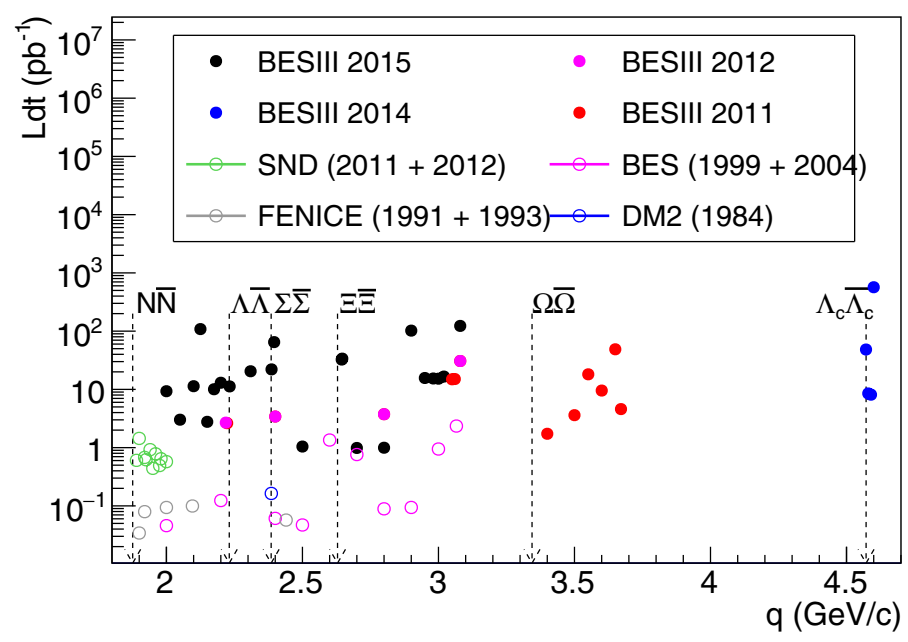

Figure 6. The integrated luminosity collected by BES III and some other $e^{+} e^{-}$ colliders as a function of CMS energy. Threshold of important baryon-antibaryon production channels are marked with vertical dashed lines.

\section{Planned upgrade of the BES III detector}

Hyperons decay weakly, i.e. they travel a finite distance before decaying. In BESIII, this distance is of the order of a few centimeters. If the decay vertex can be reconstructed with precision, the background from non-hyperonic channels can be significantly reduced. Future hyperon measurements, as well as other physics programmes in BES III, could therefore benefit from further improvements on the track- and vertex resolution. Therefore, an upgrade of the BES III detector is planned. One of the most important components of the upgraded detector is a cylindrical gas electron multiplier detector (CGEM). The detector design follows the concept of a newly installed detector in KLOE-2 [17], but deploys several innovations and dedicated features, e.g. the analog readout [18].

The CGEM detector in BES III is a joint project between INFN in Torino, Frascati and Ferarra in Italy, JGU Mainz in Germany, Uppsala University in Sweden and IHEP Beijing in China. It will be installed in the summer of 2018 and taken into operation in November, 2018.

\section{Summary}

Electromagnetic form factors provides a quantitative description of hadron structure and are fundamental observables of QCD. Baryon form factors can be studied in the time-like region by the BES III experiment. A pilot energy scan was performed in 2011-2012, resulting in new data on protonand $\Lambda$ hyperon EMFF's. A world-leading data sample has been collected in 2014-2015 which enables complete determination of $\Lambda$ and $\Lambda_{c}^{+}$form factors, precision measurements of nucleon effective form factors and first measurements of heavier single- and multistrange hyperons. A planned upgrade of the BES III detector is foreseen to 2018 where an important component is an inner tracker of a cylindrical GEM type. This will further improve the hyperon reconstructiuon in future measurements. 


\section{References}

[1] N Isgur, Excited nucleons and hadronic structure, World Scientific 403 (2000).

[2] V. Punjabi, C.F. Perdrisat and M.K. Jones, Eur. Phys. J. A 51, 79 (2015).

[3] S. Pacetti, R. Baldini-Ferroli and E.Tomasi-Gustafsson, Phys. Rept. 550-551, 1 (2015).

[4] B. Aubert et al., Phys. Rev. D 73, 012005 (2006).

[5] A.Z. Dubnickova et al., Nuovo Cim. A 109, 241 (1996).

[6] M. Ablikim et al., Nucl. Instrum. Meth. A 614 (2010) 345.

[7] V.P. Druzhinin, S.I. Eidelman, S.I. Serednyakov and E.P. Solodov, Rev. Mod. Phys. 83, 1545 (2011).

[8] M. Ablikim et al., Phys. Rev. D 91, 112004 (2015).

[9] M. Ablikim et al., Phys. Lett. B 630, 14 (2005).

[10] A. Antonelli et al., Nucl. Phys. B 517, 3 (1998).

[11] T. K. Pedlar et al., Phys. Rev. Lett. 95, 261803 (2005).

[12] T. A. Armstrong et al., Phys. Rev. Lett. 70, 1212 (1993)

[13] M. Ambrogiani et al.,Phys. Rev. D 60, 032002 (1990); M. Andreotti et al., Phys. Lett. B 559, 20 (2003).

[14] G. Bardin et al., Nucl. Phys. B 411, 3 (1994).

[15] B. Aubert et al., Phys. Rev. D 76, 092006 (2007).

[16] D. Bisello et al., Z. Phys. C 48, 23 (1990).

[17] A. Balla et al., Acta Phys.Polon.Supp. 6 no.4, 1053-1059 (2013).

[18] A. Amoroso et al., Nucl. Instrum. Meth. A 824, 515-517 (2016). 